\title{
Modeling Behaviour of Nurses in Clinical Medical Unit in University Hospital: Burnout Implications
}

\author{
Wael Rashwan \\ Technological University Dublin, wael.rashwan@tudublin.ie \\ Amr Arisha \\ Technological University Dublin, amr.arisha@tudublin.ie
}

Follow this and additional works at: https://arrow.tudublin.ie/buschmarcon

Part of the Business Administration, Management, and Operations Commons

\section{Recommended Citation}

Rashwan, W. \& Arisha, A. (2015). Modelling Behaviour of nurses in clinical medical unit in University Hospital: Burnout Implications. Proceedings of the 2015 Winter Simulation Conference, Washington, 2015.

This Article is brought to you for free and open access by the School of Marketing at ARROW@TU Dublin. It has been accepted for inclusion in Conference papers by an authorized administrator of ARROW@TU Dublin. For more information, please contact arrow.admin@tudublin.ie, aisling.coyne@tudublin.ie,gerard.connolly@tudublin.ie.

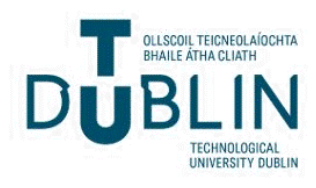




\title{
MODELING BEHAVIOR OF NURSES IN CLINICAL MEDICAL UNIT IN UNIVERSITY HOSPITAL: BURNOUT IMPLICATIONS
}

\author{
Wael Rashwan \\ Amr Arisha \\ 3S Group, College of Business, \\ Dublin Institute of Technology (DIT), \\ DUBLIN 2, IRELAND
}

\begin{abstract}
High demand of healthcare services due to changes in population demography, technological and medical advancements, budget limitations have direct effect on medical staff and medical organizations in particularly hospitals. One of the major issues confronting the healthcare system is staff behavior when they get close to 'burnout' level. This study identifies factors affecting nurses' behavior and its impact on patients experience time using system dynamics. A particular focus is given to nurses in one of the medical clinical units in one of the largest hospitals in Ireland. Armed with a comprehensive system dynamic model that revolves around the staff stresses, an examination of Skill-Mix, Work Intensity, and Time Per Activity is conducted to examine performance issues due to nurses' fatigue and burnout. Results demonstrate serious consequences on patients' experience time and service quality measures as a proportional result of the increased pressures on nurses in this unit.
\end{abstract}

\section{INTRODUCTION}

Effective planning and scheduling of human resources is a crucial challenge facing healthcare managers. Given the fact that Human Capital in healthcare organizations represents the backbone of their budget and knowledge assets, the staff planning comes as a priority. Unpredictable demand often causes a disruption in staffing pattern, which has thence adverse consequences on both cost and satisfaction levels. In healthcare service organizations, personnel scheduling has received growing attention in recent years due to its impact on work moral and patient's experience. Ineffective staffs planning decisions carry risks that wallop the quality of care and hospital performance. The staff scheduling suffers from several ramifications such as imbalance skill mix, under/over staffing, and long hours and inequitable shifts (Clark et al. 2013).

A trade-off among multiple perspectives, e.g., fairness, cost and morale has to be considered. Several data analysis studies have identified an association between staff scheduling and staff morale (Hegney, Plank, and Parker 2006). Ineffective schedules (Dunn, Wilson, and Esterman 2005), work pressure (Aiken et al. 2013), working long hours (Ann E Rogers 2008; Bae and Fabry 2013), and unfair shifts (Wilson 2002; Dunn, Wilson, and Esterman 2005) are significant factors that worsen the personal moral. A direct correlation between appropriate staffing size and lower odds of hospital-related mortality are reported in literature (Rothberg et al. 2005; Kane et al. 2007). Cost increases indirectly due to high staff turnovers (Hayes and Bonnet 2010; Jones 2005), and absenteeism (Silvestro and Silvestro 2008), and directly due to over-staffing in the ward. Therefore, optimizing staff schedule can play a key role in enhancing the performance of healthcare organizations in terms of cost, quality of care and both staff and patient satisfactions. 


\section{Rashwan and Arisha}

This paper presents a comprehensive System Dynamics model that is used to examine the nurses' behavior in a consistently demanding working environment (clinical unit in major hospital). The emphasis of the study is on the impact of nurse burnout factors on the clinical outcomes. Simulating the behavior has given an outstanding insight in how nurses response to demand fluctuations and pressures due to variability. Needless to say, the burnout adverse effect on productivity and patient experience time (PET) is clearly demonstrated in the experiments in Section 5. Background on nurses' burnout phenomenon and its implications is discussed in Section 2. While Section 3 and 4 elaborate on the model development using a thorough understanding of the factors that affect the nurses. A discussion around the three main variables (WI, TPA, and ER) is presented in Section 6.

\section{BACKGROUND}

Staff Burnout is a phenomenon that is commonly related with the work in social services such as healthcare and education organizations. Burnout can be defined as a syndrome of emotional exhaustion, depersonalization, and reduced personal accomplishments (Maslach 1982). According to (Maslach, 1998), set of identifying characteristics includes emotional exhaustion, depersonalization of treating clients, and perception of diminished personal accomplishment. Emotional exhaustion refers to a lack of energy and a feeling that one's emotional resources are used up due to excessive psychological demands

The prevalence of burnout has increased significantly, affecting 19-30\% of workers of the working global population (Finney et al. 2013). Among the medical staff, burnout has become an occupational hazard, with its rate reaching between $25 \%$ and $75 \%$ in some clinical specialties (Laschinger, Wong, and Greco 2006).

Stress in the workplace is globally considered as a risk factor for workers' health and safety. More specifically, the health care sector is a constantly changing environment, and the working conditions in hospitals are increasingly becoming demanding and stressful (Portoghese et al. 2014). In the healthcare sector, several studies report that healthcare staff are under constant of severe occupational stressors include time pressure, high workload, uncertain patients acuity, and low social support at the work (McVicar 2003). The literature indicates that burnout results from prolonged job pressure on workers. In this sense, healthcare staff are highly vulnerable to experiencing severe burnout and both mental and physical sickness (Portoghese et al. 2014). In turn, this could affect outcomes of healthcare institutions, such as the quality of care provided.

The consequences of fatigue and burnout on the performance are getting a modest attention in literature. Impact of productivity and work quality while workers feel fatigued has been compared with alcohol intoxication. Memory diminishing, slow reaction time, decreased concentration, and low motivation are among the consequences of fatigue (Blachowicz and Letizia 2006; Stimpfel, Sloane, and Aiken 2012). Rogers et al. (2004) found that hospital staff nurses' long hours can have serious implications on patient's care and safety. The likelihood of making errors is more likely to occur when hospital staff nurses work twelve or more hours. This risk increases three-time compared with eight-hour shift. In addition to other variables such as workload pressure and patient acuity increase the error. The definition of error includes apparent deviations from current standards of practice such as wrong patient, wrong medication, wrong dose, wrong route and a mistaken time. Other errors could not be discovered. There is a strong evidence that long day-shift resulted in increased fatigue of staff nurses and impaired safety and performance (Lockley et al. 2007).

\section{SYSTEM DYNAMICS}

At the end of the 1950s, Professor Jay Forrester introduced System Dynamics (SD) at the Massachusetts Institute of Technology's Sloan School of Management. He brought engineering feedback control principles and methods to management and social science situations, and then applied this approach to any complex system that exhibits dynamic behaviors over time. SD methodology attempts to simulate the system's behavior over time by representing the causal relationships between its key variables, and is 


\section{Rashwan and Arisha}

particularly suited to cases of dynamic complexity. The approach supports decision-making processes that can drive system improvement, as well as being useful in improving learning in complex systems.

Business systems exhibit two types of complexity: combinatorial complexity and dynamic complexity (Sterman 2000). Combinatorial (or detail) complexity explains how complex a problem is in terms of alternatives (Cai, Yang, and Zhang 1999; Papadimitriou and Steiglitz 1998), and can be used to represent any combinatorial problem such as staff scheduling (E. K. Burke et al. 2008). On the other hand, dynamic complexity describes the nonlinear interactions of system elements over the time and may appear even in very simple systems (Senge 2006; Sterman 2000). System complexity can be caused by the bounded rationality of decision makers or misperceptions and nonlinearity of interactions (Kampmann and Sterman 1998), and can adversely affect human decision-making processes, resulting in sub-optimal, or even unintended, results (and side effects).

Delay, accumulation and feedback loops are considered ubiquitous characteristics of healthcare systems, which is a strong argument for using system dynamics as a framework for their study (Davies and Davies 1994). A large number of elements interact in such systems, and impact on each other. Such interactions can be circular and are hard to capture, so that actions and decisions and their interrelationships may not be immediately apparent or measurable. For example, there will be a delay between when a decision is made (for instance) that a hospital expansion is needed to satisfy demand and when the expanded hospital is fully functioning. Similarly, there will be time delays and variations between when healthcare problems appear and when actions are taken to restore the system to the desired state of being able to meet demand. Furthermore, the existence of nonlinear relationships increases the difficulty of predicting healthcare systems' behaviors accurately and complicates decision-making processes. For example, the relationship between patients' admission waiting times and lengths of stay (LOS) is nonlinear. Chalfin et al. (2007) show that if a patient is admitted immediately, it is likely that their LOS will be short - but, if they have to wait a long time to be admitted, the LOS is likely to be significantly longer. And the patient's state may worsen considerably while waiting for medication, particularly if they are elderly.

SD offers a methodology that can help businesses and government institutions to develop a strategy and analyze policy interventions by modeling causal relationships and feedback systems (Sweetser 1999). The method has been applied to model such strategic aspects of policy and national issues in care systems as patient's pathways (Monefeldt, Lane, and Rosenhead 2000) and planning care for the elderly (Wolstenholme 1999; Walker and Haslett 2001; Desai et al. 2008). A system dynamics model has been developed for healthcare in Alberta, Canada (Cooke et al. 2007) to evaluate health policies for emergency services. In the same hospital, Robertson, Bloom and Duckett (2012) has developed an interactive model for planning the workforce for healthcare based on system dynamics. System dynamics also has been used for mapping of acute patient flows for NHS in England (Lane and Husemann 2007). Masnick and McDonnell (2010) applied system dynamics modeling to link clinical workforce requirements to the clinical workforce workload. Their model produced the broad of healthcare that helps human resource planners to improve the decision-making process. The consequences of policies intended to restore the performance of healthcare systems to their desired state may be disappointing: explanations may include staff resistance to new policies and counter-intuitive behavior on the part of the policymakers. Simulation and modeling can be useful and flexible tools to tackle several of these concerns and to contribute towards improved health system performance and thus better health care provision.

\section{MODEL CONCEPTUALISATION}

System Dynamics modeling is used in this study in order to integrate the patient's flow in a medical clinical unit in university hospital and incorporate nurse behavior in response to workload stresses. The model seeks to explain the implications of constant workload stresses on nurses' performance during their shifts. Theoretical foundations from literature and evidences from field study are used during the modeling stages. There are three components that the model aims to address: 


\section{Rashwan and Arisha}

1. Patient flow element that tracks the physical flow of patients.

2. Medical Unit capacity component - models the management policies regarding to staff size, bed capacity, staff scheduling, and staff skill-mix. This component considers different types of human resources with different experience levels such as senior nurses, assistant nurses, senior physician (registrar), junior physician (SHO) and consultant physician.

3. Staff Behavior component that models how the staff deals with workload stresses. This study has a particular focus on the response of nurses to demand capacity imbalance and work pressures.

\subsection{The Road to Nurses Burnout}

Since the healthcare service requires physical presence of medical staff and patients, this creates inevitable sensitivity to demand-capacity gaps. Owning the fact that the service buffer is not a valid option either economically or strategically, the management of healthcare services has to find solutions to demand uncertainty for patients whether in prolonged waiting time, extended LOS and compromise in the service quality, and/or opportunities for unintended errors. On the other side, it creates a workload pressure on the healthcare staff members which often causes untoward consequences on their health and safety (Portoghese et al. 2014). The literature indicates that staff burnout is a result of prolonged job pressure on workers. In this sense, healthcare staffs are highly vulnerable to experiencing severe burnout and both mental and physical sickness. This will lead to issues at different levels of the healthcare organizations.

Medical teams tend to perceive the workload pressures when they noticed a deviation between the intended work to be completed within a period of time and the actual work done in the same span of time. Under constant work stresses, the medical staff struggles to cope with the increasing demand during their working hours while sustaining the anticipated quality of care. Staff satisfaction and quality of care is determined by how the staff adopted to respond to work pressures. Since workload stresses have direct consequences on quality of care and staff satisfaction, it becomes essential to understand the behavior of staff when they work under these stresses that can easily lead to burnout. On one side, the imbalance in service capacity and demand eventually translate into difficulties.

\subsubsection{Workload Pressures}

The workload on nurses at any particular moment of time is composed of patients who are undergoing their treatment and also the patients who are currently waiting to be seen. Following Little's law, the average patient's experience time (PET) is the ratio between workload and discharge rate. The discharge rate is determined by the effective staff capacity and treatment time per patient. Figure 1 presenting a simplified causal loop diagram of workload pressure in the MCU.

The nurse workload pressure is the ratio between the actual nurse-to-patient ratio and the standard nurse-to-patient ratio. Workload pressure value is less than one implies overstaffing while if it is greater than 1, this indicates that the unit is stretched with and stresses on nurses will take place. Since nurses have different psychological attributes, perceptions, and expectations, the workload pressure effect will strike after a delay in time subject to their agility. Extremely high workload pressure can literally prevent the unit from effective treatment of patients within reasonable PET given the unit nurse/doctors capacity, working hours, time allocated for patient treatment, and support received from other units such as laboratories and radiology. On the other hand, the hospital managers are under pressure to maintain the target PET by healthcare executives. This type of pressure is referred to as a desired service level pressure that can be mathematically defined by the ratio between the desired PET and the actual PET.

In order to alleviate some of the work pressure, staff capacity increase and/or process redesigning can lead to feasible solutions. Nevertheless and in the healthcare context, the reaction from the senior management to high workload pressure to these solution is slow and inflexible (Rogelio Oliva and Sterman 2010). Time to recruit new staff including negotiation, procedure to follow to have a case to 


\section{Rashwan and Arisha}

change the process, training new staff or induction for part-time staff, and delay in responses from authority can cause more delay in the action to the workload pressures. Hence, the unit waiting for a response from management to increase the capacity will see the nurses adjusting to the high pressures at work by different behavioral approaches. Disregardng capacity expansion issue was well explained by Oliva and Sterman (2001); two possible responses can result from staff in order to reduce the work pressure: (1) working harder and this will swiftly lead to a burnout in a shorter time or (2) reducing the time allocated to the customer and equally the quality. These possible reactions have been validated through nurses' interviews and focus group.

\subsubsection{Work Intensity (WI)}

Nurses can respond to high perceived workload pressure and throughput (desired PET) pressure by working hard and increasing work intensity. This response entails that they increase their efforts, concentration, engagement, and cutting short their number and duration of breaks. They can also working overtime in order to fill the demand. Increasing work intensity creates a balancing loop by increasing the effective nurse capacity and in turn alleviates the workload pressures (balanced feedback loop B3 and B4). Working hard increases the discharge rate (productivity) of the patients due to the increase in the effective nurse capacity and this will contribute to the reduction of the workload pressures (balanced feedback loop B4). During the interview with the nurses in the hospital, they indicated that they often implement this practice but they flagged that they feel worn out quickly due to this.

\subsubsection{Treatment Time Per Activity (TPA) Adjustment}

Research reported that nurses sometimes tend to reduce time allocated to some activities in order to cope with the high demand imposed on them (Kalisch, Landstrom, and Hinshaw 2009). Nurses can also react to high-perceived workload pressures and desired PET by adjusting the time allocated per patient and reducing the care attention thereof. Due to the acute nature of the healthcare service, adjusting the treatment time per patient is often not an option. This also can be extended to reduce the administration work associated with the patients such as documentation work, data entry and witting reports, which is important element in the process. Reducing the treatment time per patient effectively speed up the discharge rate which decreases workload and eventually reduce the workload pressures (loop B2 and B5). Similarly, reducing the administration work increases the effective nurse capacity, thus eventually mitigates the pressure (loop B6). This reaction creates two balancing feedback mechanisms that limit both workload pressure and desired PET pressure.

\subsection{Burnout Implications}

Healthcare staff who are under constant of severe occupational stressors such as time pressure, high workload, and uncertain patients acuity are vulnerable to experiencing severe burnout and both mental and concentration, and low motivation are among the consequences of fatigue (Blachowicz and Letizia 2006; Stimpfel, Sloane, and Aiken 2012). Burnout results from a combination of factors including: prolonged job pressure on workers, stresses due to demand fluctuations, short in experienced staff, and/or work environment. Needless to say that staff burnout is one of the main factors that deteriorate the organization morale and equally the productivity rate (Homer 1985). The nurses' burnout process begins when the staff attempts to fulfill the high workload by working intensively and for long hours, which increases the stress exposure that drains their energy. Therefore, draining the nurses' energy level reduces the nurses' productivity, thus reducing the effective nurse capacity and increasing the pressure. In turn it depletes their energy further and increasing the opportunity of nurses' burnout to happen. The unintended consequences create a reinforcement feedback loop (R1). Growing the staff fatigue (depleted energy) increases the exposure to continuous work stress and the energy depleted more rapidly creating a 


\section{Rashwan and Arisha}

reinforcement loop R2 (frustration-exhaustion). Unlike, high energy level enhances individual moral and satisfaction, which helps to recover rapidly from temporarily fatigue (loop R3) from rapidly from burnout. The failure to fulfill the desired goal (desired PET) is an additional stressor that creates a pressure from the management, which pushes staff to works hard in order to achieve the desired PET and in turn undermine the energy. Recovery from burnout can be achieved by reducing workload and less management expectations, which reduce the staff energy draining. Also, shift breaks and days off helps to recover the energy decay during the day and the week. Without adequate breaks to recover the energy will deplete faster. However, when the unit is under work stress, the nurses may not take adequate breaks during their duty hours or they cannot find the staff coverage at the break time. Thus, the inadequacy of shifts breaks delay the fatigue recovery and increases the exhaustion. This behavior creates a reinforcement mechanism loop (R4). Another implication of burnout is the likelihood of random absenteeism is associated with low energy level. Increasing the absenteeism reduces number of staff nurses available to work, thus reducing the effective productivity that in turn raises the workload pressure and eventually the fatigue. This unintended implication is depicted by reinforcement loop R5. The impact of unplanned staff absenteeism continues for a while (time delay) until the nurse manger can find a replacement.

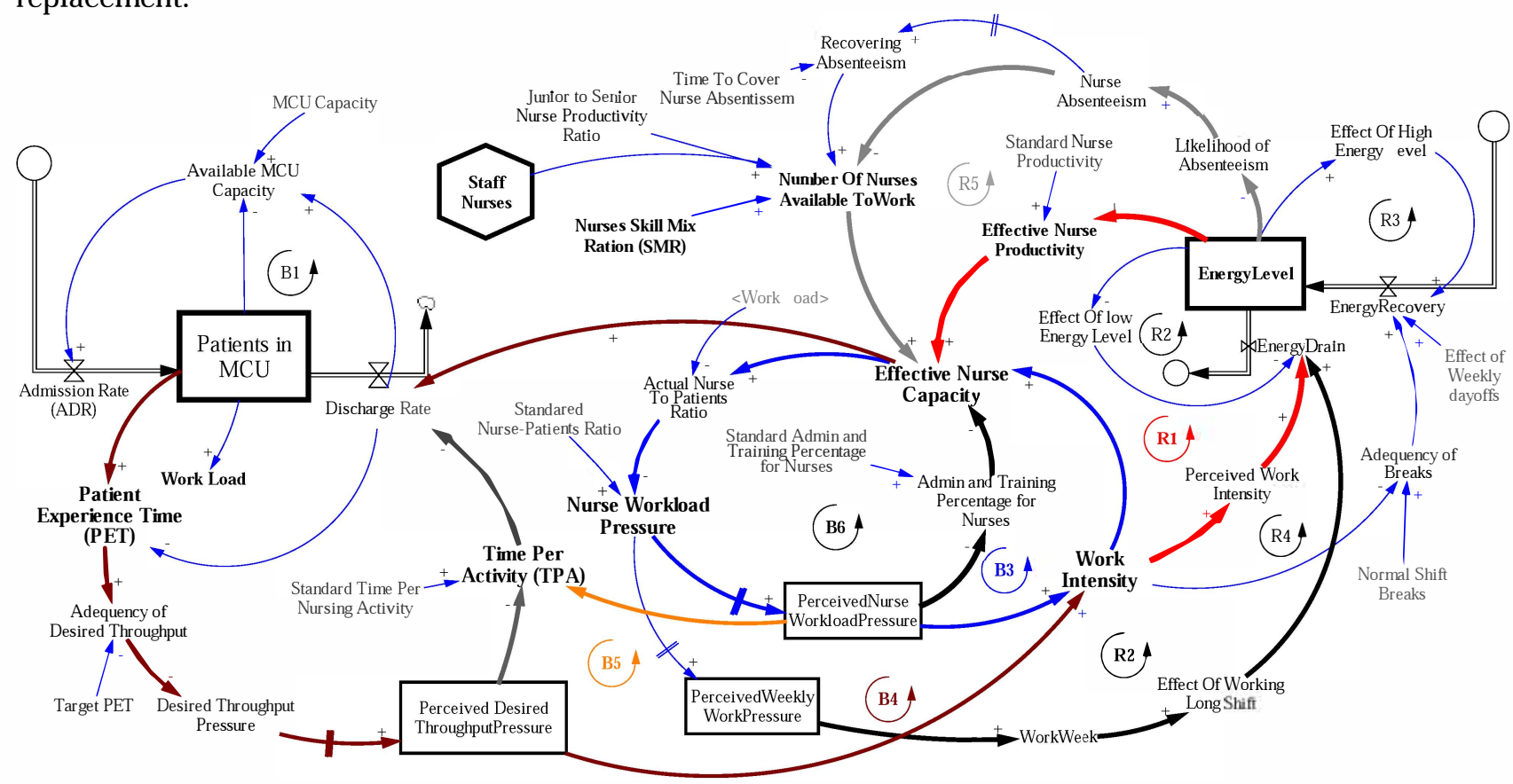

Figure 1: Causal loop diagram for the responses of staff nurses to workload pressure.

\subsection{Model Validation}

To reduce the model's development cycle time and to increase the confidence in its results, verification and validation were carried out throughout the development phases of the project (Roberts 2011). Several model tests - mainly structure and behavior tests - were applied to the model as suggested in SD literature(Barlas, 1989, 1996; Sterman, 2000). The model structure was discussed and verified with MCU team at different stages to ensure its consistency. Integration time step $(\boldsymbol{d t})$ was set to one-half the smallest shortest time parameter in the model that equals to 1 minute. The historical data collected for six months are used validate the model results. Table 1 provides a comparison of the actual and simulated data. The comparison reveals the percentage of discrepancy is to some extent within the acceptable ranges 
Table 1: Validation of simulation model.

\begin{tabular}{lccc}
\hline & $\begin{array}{c}\text { Patient Experience Time } \\
\text { (PET) }\end{array}$ & $\begin{array}{c}\text { Percentage of } \\
\text { Admission to MCU }\end{array}$ & $\begin{array}{c}\text { Percentage of } \\
\text { Admission to } \\
\text { Ward }\end{array}$ \\
\hline $\begin{array}{l}\text { \% Of deviation from } \\
\text { actual values }\end{array}$ & $-9 \%$ & $5 \%$ & $8 \%$ \\
Simulated & 6.2 & $21 \%$ & $17 \%$ \\
\hline
\end{tabular}

\section{ANALYSIS}

The developed model for the study presents a combination of complex dynamic sub-modules that are characterized by stochastic and non-linear features. This makes a hard task for interpretation and analysis of the model outcomes. Sensitivity analysis can be a useful technique to use. It helps to vary model parameters while observing how behavior will impact on the performance measures. Sensitivity analysis helps for model testing, learning, and validation (Moxnes 2005). System dynamics allows experimenting and support sensitivity analysis for selected hypothetical values of the model parameters. The analysis requested from the management has been classified into three sets of experiments. Each experiment has two key settings; one is to examine the model behavior assuming the burnout has not taken place yet (no burnout; NBO) on the nurses' behavior, whilst the second case considers the burnout is on (with burnout; WBO).

Three performance measures are used to assess the implications of changing skill-mix ratio (SMR): average patient experience time (PET), percentage of administrative work reduction (AWR) and percentage of admission (PAD) by MCU. . PET is the Length of Stay (LOS) of patients in the unit; PAD is the percentage of the presenting medical ED patients that are admitted by the MCU, while AWR calculates the percentage of admission word reduction as a response to high workload and service level pressures as discussed in section 4.2. During the model development process it becomes evident the PET is the key performance measure for the management of the MCU.

\subsection{Skill-Mix Implications}

Mismanagement of nurse shift schedules carries risks and can lead to fatigue and stresses that eventually impacts negatively on service quality. Imbalanced skill-mix shift is one of the characteristics of weak scheduling decisions (Clark et al. 2013). The first set of experiments recommended by the management is to examine changing the shift skill-mix ratio (SMR) for nurses and its effect on the unit performance. SMR is apparently critical call for managers because it matters in assigning senior staff with junior staff in particular shifts. Striking the right balance of the mix while maintaining the workload as fair as possible is a challenging task of the unit manager. Not to mention the unforeseen events of staff sickness, epidemic infections, or personal holiday requests that feature extra burden on managers to optimize the resources and the productivity of the unit. The standard agreed clinical skill-mix ratio in the units is four experienced nurses to one junior nurse. The values of the performance measures for this experiment are shown in Figure 2.

In this model, SMR can take a value from 0 to 1 , where 0 means that all staff nurses in a shift are not experienced and 1 means that they are all senior experienced team. The model dynamically simulates the behavior of the levels of SMR between 0 and 1, with an increment of 0.1 in every set of runs. Results reveal the high implications of SMR on the performance measures. The baseline value of SMR is 0.75 . In both cases, NBO and WBO, the PET and percentage of administrative work reduction are increasing as the percentage of experienced nurses increases, whilst the PET decreases significantly (Figure 2a). If the all staff nurses are experienced (high skill grade), the PET dramatically decreased by more than $63 \%$ in the case NBO and by more than $100 \%$ in case WBO. Similarly, Figure $2 \mathrm{~b}$ show that the PAD has increased from $16 \%$ to reach $23 \%$ when no burnout and from $12 \%$ to $25 \%$ when nurse burned-out. 


\section{Rashwan and Arisha}

Imbalanced case mix (i.e., low SMR) has greater impact on the nurses' productivity that creates additional pressure on the staff nurses to work hard in order to meet the demand imposed on them by reducing their administrative work and also in reducing the shift breaks length. When SMR is 0 (all staff nurse are junior), the amount of admin work reduction is $38 \%$ and $39 \%$ in case of NBO and WBO respectively (Figure $2 \mathrm{~b}$ ). These percentages reach to $12 \%$ and $25 \%$ when all nurses are experienced for NBO and WBO respectively. When the nurses are burned-out, AWR still records a high value even though all nurses in the shift are seniors. This can be explained by looking at the causal loop B6 in Figure 1 where the continuous workload pressure impacts negatively on the productivity of nurses and they respond by increasing AWR which in turn reduce the energy level.
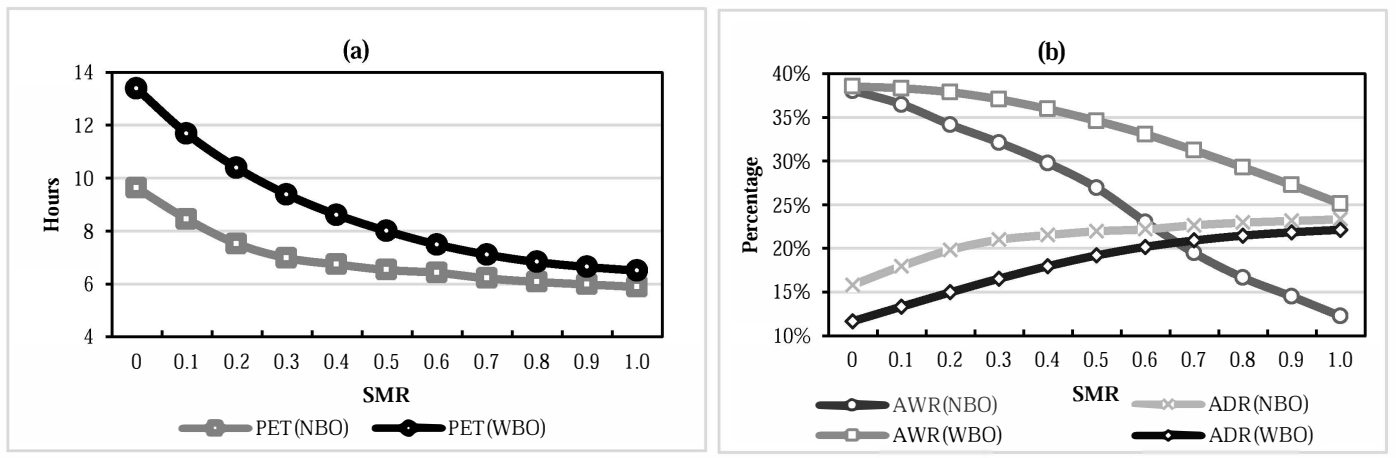

Figure 2: Sensitivity analysis experiment for the SMR implications.

\subsection{Work Intensity}

Work intensity (WI) is positively correlated with indicators of work demands long working hours, job stress and high perceived workload (R. J. Burke, Singh, and Fiksenbaum 2010). The response of staff nurses to high workload and service level pressures are also examined. Figure 3 demonstrates the sensitivity analysis results of changing the work intensity of the nurses as a reaction to the increase in workload pressures. WI increases productivity of staff nurses because they reduce length of their breaks, show more dedications, reduce their admin work, and/or sometimes work overtime voluntarily. The Implications of WI is tested over nine levels $0 \%-30 \%$ by $5 \%$ increment factor.

WI has a significant positive effect on the three performance measures. When the WI is not allowed (i.e., 0\%), due to the burnout implications, the PAD increases by $16.6 \%$ (Figure $3 \mathrm{~b}$ ) and the PET (Figure 3a) also increases by about 29\% comparing with NBO. Likewise, when the maximum WI reaches 30\%, the effect of fatigue and burnout significantly result in a $14.3 \%$ (Figure $3 \mathrm{~b}$ ) reduction in PAD a dramatic jump in PET by 53.6\% (Figure 3b).
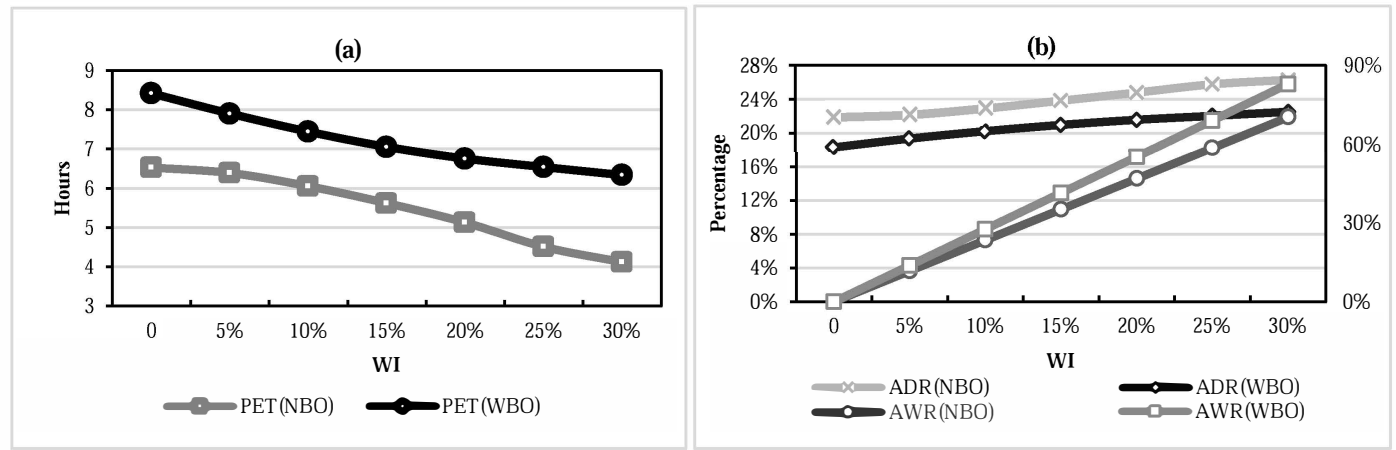

Figure 3: Sensitivity analysis experiment for the Work Intensity behavior (WI) implications. 


\section{Rashwan and Arisha}

Interestingly, working hard has series of unexpected consequences. For example, results show that at WI equal to nearly $30 \%$, a noticeable reduction in the admin work by $83 \%$ is recorded. First, consistent WI induces fatigue and burnout promptly and reduces productivity and concentration. It also increases absenteeism and medical errors. Second, continuous pattern of WI will reduce administrative work of patients' records and this can cause a slowdown in other activities related to the treatment or error that can lead to worse consequences. This can also easily interrupt training and improvement programs. From interviews with nurses, cancel their breaks or shorten them creates a negative effect on their mental health and on the long term can lean to breakdown. In fact, the unintended consequences of this behavior on the quality of care (i.e., medical errors) are important than what this model may reveal. Recording errors (medical or administrative) seem to be a serious problem in most of the healthcare systems. Staff never record recoverable errors in order to keep team spirit and increase work morale.

\subsection{Time per Activity}

Research reported that nurses sometimes tend to reduce time allocated to some activities in order to cope with the high demand imposed on them (Kalisch, Landstrom, and Hinshaw 2009). The omission of care is translated into abbreviating the care, delays the care or may simply omit the care (e.g. not developing the patient's plan of care). This behavior is reflected into the model through adjusting (shortening) the time allocated to nursing activity in order to speed up the process.

In the model (i.e., balanced feedback loops B2 and B5), the TPA is dynamically adjusted based on the perceived workload pressure and desired service level pressure (i.e., desired PET). This experiment explores the impact of the adjusting TPA from the standard values, as it is very difficult to quantify numerically due to lack of data and the difficulty to collect such information. The model behavior is simulated under reasonable reduction of the standard TPA ranging from $0 \%$ to $16 \%$ by $2 \%$ increment. Figure 4 shows the summary of 18 experiments. As might have been expected, reduction in the TPA has a considerable impact on performance measures. A reduction of PET and an increase in the ADR takes place when the TPA is reduced by $16 \%$ comparing to the standard TPA (i.e., without reduction). The PET dropped by more than $38 \%$ (2.4 hours reduction) in NBO case and by $31.9 \%$ (3.1 hours reduction) in WBO case (Figure $4 \mathrm{a}$ ), whilst the ADR is increased by $17 \%$ and $31.7 \%$ (Figure $4 \mathrm{~b}$ ) in the cases of NBO and WBO respectively. For WBO, the PET reduced by 3.1 hours when the standard TPA lowered by $16 \%$ with average slope 0.19375 hours (i.e., 3.1/16). Thus reducing the standard TPA by $1 \%$ is associated with a reduction in the PET by 0.19375 hours (11.6 minutes). Likewise, ADR has increased considerably increased by $6 \%$ with average rate $0.375 \%$ per $1 \%$ reduction in the TPA.

However, this behavior has complicated unintended consequences for losing the healthcare standard that leads to undermine the quality. Patient safety is at the center to the practice of nursing, and is influenced by errors of care omission. The consequences of missed nursing care to as human behavior to respond to the workload pressure carries risks to patient safety. Unfortunately, the possible deterioration on the quality of care and medication errors is not considered in this study.

\section{CONCLUSION}

Balance of medical staff workload is a critical issue that has a significant impact on the paradigm of healthcare system internationally. Medical staff burnout consequences vary from error in diagnosis, operational issues, complexity in scheduling, failure in hospital redesign process and unforeseen patients experience.

This study examines a clinical unit in a large hospital in Ireland in order to find the impact of nurses' behaviors when they experience burnout level on unit performance measures. Working with the nurses and the management team of the unit, comprehensive System Dynamics model was developed to encompass the factors that may contribute to the burnout phenomenon and also the relationship between 

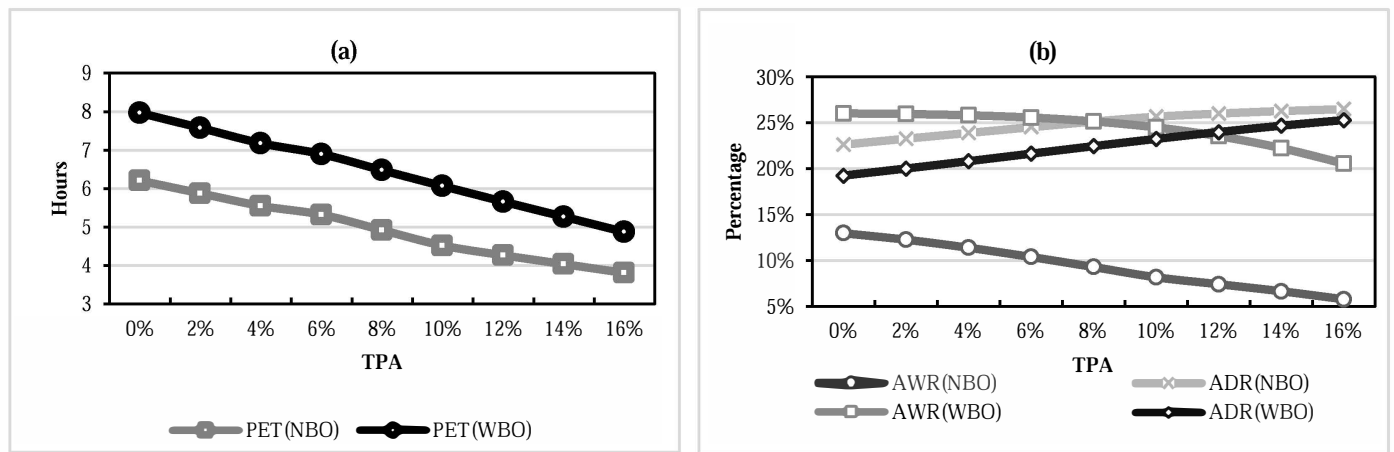

Figure 4: Sensitivity analysis experiment for the reducing time per nursing activity behavior (TPA) implications.

these factors and the performance measures. Data collected from semi-structure interviews with nurses and Focus Groups have helped to estimate few parameters while the remaining of the ratios was used based on the literature recommendations. Four main elements of the burnout phenomenon received extra attention in this study: Skill-Mix (SM), Work Intensity (WI), Time Per Activity (TPA), and Extra Resources (ER). Nurses react to the burnout effect by adjusting their behaviors to cope with the fatigue, stresses and workload pressures. This reaction can be described as a natural defense to survive the tide.

Results show that the imbalanced skill-mix has greater impact on the nurses' productivity that creates additional pressure on the staff nurses, which eventually leads burnout. The work intensity behavior experiment indicates that working hard mitigate the workload pressure for temporarily and eventually depletes the energy nurses' energy level which has dramatic unintended consequences. Finally, reduction the time per nursing activity associated with significant reduction in the workload pressure, this behavior has complicated unintended consequences for losing the healthcare standard that leads to undermine the quality.

The study recommends the use of smart scheduling system to plan the nurses' shifts and workload and that the organization management has to be careful of the impact of the staff burnout on performance. Demand fluctuation will require better planning especially with limited capacity and cost reduction policies.

\section{REFERENCES}

Aiken, Linda H., Douglas M. Sloane, Luk Bruyneel, Koen Van den Heede, and Walter Sermeus. 2013. "Nurses' Reports of Working Conditions and Hospital Quality of Care in 12 Countries in Europe." International Journal of Nursing Studies 50: 143-153.

Bae, Sung-Heui, and Donna Fabry. 2013. "Assessing the Relationships between Nurse Work Hours/overtime and Nurse and Patient Outcomes: Systematic Literature Review.” Nursing Outlook 62(2):183-65.

Blachowicz, Ewa, and MariJo Letizia. 2006. "The Challenges of Shift Work." Medsurg Nursing : Official Journal of the Academy of Medical-Surgical Nurses 15 (5) : 274-80.

Burke, E K, Timothy Curtois, Gerhard Post, Rong Qu, and Bart Veltman. 2008. "A Hybrid Heuristic Ordering and Variable Neighbourhood Search for the Nurse Rostering Problem.” European Journal of Operational Research 188 (2) (July): 330-341.

Burke, Ronald J., Parbudyal Singh, and Lisa Fiksenbaum. 2010. "Work Intensity: Potential Antecedents and Consequences." Personnel Review 39 (3): 347-360.

Cai, M.C., X.G. Yang, and J.Z. Zhang. 1999. "The Complexity Analysis of the Inverse Center Location Problem." Journal of Global Optimization 15 (2) (September 1): 213-218.

Chalfin, Donald B, Stephen Trzeciak, Antonios Likourezos, Brigitte M Baumann, and R Phillip Dellinger. 2007. "Impact of Delayed Transfer of Critically Ill Patients from the Emergency Department to the Intensive Care Unit." Critical Care Medicine 35 (6): 1477-83. 


\section{Rashwan and Arisha}

Clark, Alistair, Pam Moule, Annie Topping, and Martin Serpell. 2013. "Rescheduling Nursing Shifts: Scoping the Challenge and Examining the Potential of Mathematical Model Based Tools." Journal of Nursing Management 23(4):411-20.

Cooke, David, Huiming Yang, Gil Curry, Paul Rogers, Thomas Rohleder, Robert Lee, and David Strong. 2007. "Introducing System Dynamics Modeling to Health Care in Alberta." In Proceedings of the 2007 System Dynamics Conference.

Davies, R, and HTO Davies. 1994. "Modelling Patient Flows and Resource Provision in Health Systems." Omega 22 (2): 123-131.

Desai, Mitul Shivam, Marion Louise Penn, Sally Brailsford, and Max Chipulu. 2008. "Modelling of Hampshire Adult Services—gearing up for Future Demands." Health Care Management Science 11 (2) (24): 167-176.

Dunn, Sandra, Brenda Wilson, and Adrian Esterman. 2005. "Perceptions of Working as a Nurse in an Acute Care Setting." Journal of Nursing Management 13: 22-31.

Finney, Caitlin, Erene Stergiopoulos, Jennifer Hensel, Sarah Bonato, and Carolyn S Dewa. 2013. "Organizational Stressors Associated with Job Stress and Burnout in Correctional Officers: A Systematic Review." BMC Public Healthublic Health 13 (1): 1-13.

Hayes, Bronwyn, and Ann Bonnet. 2010. "Job Satisfaction, Stress and Burnout Associated with Haemodialysis Nursing: A Review of Literature.” Journal of Renal Care 36: 174-179.

Hegney, Desley, Ashley Plank, and Victoria Parker. 2006. "Extrinsic and Intrinsic Work Values: Their Impact on Job Satisfaction in Nursing." Journal of Nursing Management 14: 271-281.

Homer, Jack B. 1985. "Worker Burnout: A Dynamic Model with Implications for Prevention and Control." System Dynamics Review 1 (1): 42-62.

Jones, Cheryl Bland. 2005. "The Costs of Nurse Turnover, Part 2: Application of the Nursing Turnover Cost Calculation Methodology." The Journal of Nursing Administration 35: 41-49.

Kalisch, Beatrice J., Gay L. Landstrom, and Ada Sue Hinshaw. 2009. "Missed Nursing Care: A Concept Analysis." Journal of Advanced Nursing 65 (7): 1509-1517.

Kampmann, C, and JD Sterman. 1998. "Feedback Complexity, Bounded Rationality, and Market Dynamics." Memo D-4802, System Dynamics Group. Massachusetts Institute of Technology.

Kane, Robert L, Tatyana A Shamliyan, Christine Mueller, Sue Duval, and Timothy J Wilt. 2007. "The Association of Registered Nurse Staffing Levels and Patient Outcomes: Systematic Review and MetaAnalysis." Medical Care 45(12): 1195-1204.

Lane, DC, and E Husemann. 2007. "System Dynamics Mapping of Acute Patient Flows." Journal of the Operational RESEARCH SOCIETY 59(2), 213-224.

Laschinger, Heather K Spence, Carol A Wong, and Paula Greco. 2006. "The Impact of Staff Nurse Empowerment on Person-Job Fit and Work Engagement/burnout." Nursing Administration Quarterly 30 (4) (January): 358-367.

Lockley, Steven W, Laura K Barger, Najib T Ayas, Jeffrey M Rothschild, Charles a Czeisler, and Christopher P Landrigan. 2007. "Effects of Health Care Provider Work Hours and Sleep Deprivation on Safety and Performance." Joint Commission Journal on Quality and Patient Safety 33 (supplement1): 7-18.

Maslach, C. 1982. "Understanding Burnout Definitional Issues in Analyzing a Complex Phenomenon." Job Stress and Burnout In W.S. Pa: 29-40.

Masnick, Keith, and Geoff McDonnell. 2010. "A Model Linking Clinical Workforce Skill Mix Planning to Health and Health Care Dynamics." Human Resources for Health 8(1): 11.

McVicar, Andrew. 2003. "Workplace Stress in Nursing: A Literature Review." Journal of Advanced Nursing 44 (6): 633-642.

Monefeldt, C, DC Lane, and JV Rosenhead. 2000. "Looking in the Wrong Place for Healthcare Improvements: A System Dynamics Study of an Accident and Emergency Department." Journal of the Operational Reserach Society 51 (5): 518-531.

Moxnes, Erling. 2005. "Policy Sensitivity Analysis: Simple versus Complex Fishery Models." System Dynamics Review 21 (2): 123-145.

Oliva, R., and JD Sterman. 2001. "Cutting Corners and Working Overtime: Quality Erosion in the Service Industry.” Management Science 47(7), 894-914. 


\section{Rashwan and Arisha}

Oliva, Rogelio, and John D Sterman. 2010. "Death Spirals and Virtuous Cycles: Human Resource Dynamics in Knowledge-Based." Handbook of Service Science. Springer US, 321-358.

Papadimitriou, Christos H., and Kenneth Steiglitz. 1998. Combinatorial Optimization: Algorithms and Complexity. Courier Corporation.

Portoghese, Igor, Maura Galletta, Rosa Cristina Coppola, Gabriele Finco, and Marcello Campagna. 2014. "Burnout and Workload Among Health Care Workers: The Moderating Role of Job Control." Safety and Health at Work 5 (3): 152-157.

Robertson, Andrea, Judy Bloom, and Stephen Duckett. 2012. "Development of an Interactive Model for Planning the Care Workforce for Alberta: Case Study." Human Resources for Health 10(1):22.

Rogers, A. E., W.-T. Hwang, L. D. Scott, L. H. Aiken, and D. F. Dinges. 2004. "The Working Hours Of Hospital Staff Nurses And Patient Safety." Health Affairs 23(4), 202-212.

Rogers, Ann E. 2008. "The Effects of Fatigue and Sleepiness on Nurse Performance and Patient Safety." In Patient Safety and Quality: An Evidence-Based. Handbook for Nurses, 509-545.

Rothberg, Michael B, Ivo Abraham, Peter K Lindenauer, and David N Rose. 2005. "Improving Nurse-toPatient Staffing Ratios as a Cost-Effective Safety Intervention.” Medical Care 43: 785-791.

Senge, Peter M. 2006. The Fifth Discipline: The Art \& Practice of The Learning Organization. Doubleday.

Silvestro, Rhian, and Claudio Silvestro. 2008. "Towards a Model of Strategic Roster Planning and Control: An Empirical Study of Nurse Rostering Practices in the UK National Health Service." Health Services Management Research, 21(2), 93-105.

Sterman, John D. 2000. Business Dynamics: Systems Thinking and Modeling for a Complex World. McGrawHill/Irwin.

Stimpfel, Amy Witkoski, Douglas M Sloane, and Linda H Aiken. 2012. "The Longer the Shifts for Hospital Nurses, the Higher the Levels of Burnout and Patient Dissatisfaction." Health Affairs 31 (11): 2501-9.

Sweetser, Al. 1999. "A Comparison of System Dynamics (SD) and Discrete Event Simulation (DES)." In System Dynamic Society.

Walker, Beverly, and Tim Haslett. 2001. "System Dynamics and Action Research in Aged Care." Australian Health Review 24 (1): 183.

Wilson, Janet L. 2002. "The Impact of Shift Patterns on Healthcare Professionals." Journal of Nursing Management 10(4): 211-219.

Wolstenholme, E. 1999. "A Patient Flow Perspective of UK Health Services: Exploring the Case for New 'intermediate Care' Initiatives.” System Dynamics Review 15 (3): 253-271.

\section{AUTHOR BIOGRAPHIES}

WAEL RASHWAN, MSc. is a researcher in 3S Group (A specialized research center in systems simulation and optimization) based in College of Business - Dublin Institute of Technology (DIT)). He joined the 3S Group in 2013. He successfully completed his B.Sc. and M.Sc. degree in Operations Research form Cairo University. Wael has extensive experience working in IT Industry. His research interests include: Modeling and Simulation, Optimization, Machine Learning, Game Theory and Probability Collectives. His email address iswael.rashwan@mydit.ie

AMR ARISHA, Ph.D. is the Director of the 3S Group and the Head of International Business Department at College of Business, Dublin Institute of Technology (DIT). He Joined DIT in 2005, he received his PhD in Industrial Engineering from Dublin City University (DCU). Intel-Ireland has sponsored his research from 2000 - 2005. His research interests include analysis and optimization of complex dynamic systems in manufacturing and service industries. He is a member in IIE, IMECH, IEI, ESE, ORS, IEEE and ASME and Chief Examiner at MII. His e-mail address is amr.arisha@dit.ie. 